\title{
Review Paper on Identifying Features in Opinion Mining via Intrinsic and Extrinsic Domain Relevance
}

\author{
Swapnil More ${ }^{1}$, Preeti Kale ${ }^{2}$ \\ ${ }^{1}$ Department of Information Technology, Pune, India \\ ${ }^{2}$ Professor, Department of Information Technology, Pune, India
}

\begin{abstract}
In this paper, we have planned to propose a complete unique technique to spot opinion features from on-line reviews by exploiting the two distinct opinion feature statistics across two corpora, one domain-specific corpus (i.e., the given review corpus) and one domain-independent corpus (i.e., the contrastive corpus). We capture this inequality via domain connection (DR) that characterizes the connection of a term to a text assortment. Initial list extraction of candidate opinion options is done from domain review corpus by following the grammar dependence rules. For every extracted candidate feature, we can estimate its intrinsic-domain connection (IDR) and extrinsic-domain connection (EDR) scores on the domain-dependent and domain-independent corpora, severally. The aim of document-level (sentence-level) opinion mining is to classify the general judgment or sentiment expressed in a personal review document. Thus, on the basis of candidate feature, the interval threshold can be used for intrinsic and extrinsic domain connection criterion. Evaluations conducted on two real-world review domains demonstrate the effectiveness of our projected IEDR approach in distinguishing opinion options.
\end{abstract}

Keywords: Information search and retrieval, IDR, EDR, IEDR opinion mining, opinion feature

\section{Introduction}

This technique is to identify opinion features from user opinions on any product. These opinions are important role in sale of the product. This work is to extract opinion features from user opinions to identify on which feature users are going to do opinion. There are number of techniques for the identification of these features but, they are operated on a single corpus and ignore nontrivial distribution in word. This work discusses a novel method for mining features in user opinions from two types of corpus one is domain dependent and other is domain independent. A supervised learning approach [2] [3] works well in given domain only but cant retain in other domain. Unsupervised approach [4] [5] [6] will apply some of the syntactic rules for opinion feature identification. Topic modeling approach [7] [8] is to mine generic topics.

One solution is the selection of domain independent corpus. Such that frequency of feature in user review is more in domain dependent corpus than the domain independent corpus. Let us consider one example containing feature battery. This feature may be present in both mobile domain and vehicle domain. The frequency of feature is high in mobile domain and relatively less in vehicle domain. The feature extraction from two domains is better achieved using novel technique. In novel technique domain relevance score is measured for each domain dependent and domain independent corpus [10] [11]. The measurement of domain relevance score on domain dependent score is termed as intrinsic domain relevance, in other case domain relevance score on domain independent corpus is termed as extrinsic domain relevance. The application of Intrinsic Extrinsic domain relevance on results of previous step yields accurate opinion features from user reviews.
Rules which are not in proper structure are unable to work well on colloquial real-life reviews. Topic modeling approaches can extract coarse-grained and generic topics, which are actually semantic feature clusters of the precise features commented on explicitly in reviews [3].

Without taking into consideration the distributional characteristics of opinion features in another different corpus presented corpus statistics techniques attempt to extract opinion features by extracting mining statistical patterns [5] of feature terms only in the given review corpus.

Proposed method is stated as follows:

1) To produce a list of candidate features from the given domain review corpus, a number of syntactic dependence set of laws are used.

2) We compute domain relevance score for each documented feature candidate with respect to the domainspecific and domain independent corpora. Domain relevance score of domain-specific corpora is known as intrinsic-domain relevance (IDR) score and the domain relevance score of domain independent corpora is known as extrinsic domain relevance (EDR) score

3) Finally, candidate features with low IDR scores and high EDR scores are pruned. We, thus, call this interval thresholding the intrinsic and extrinsic domain relevance (IEDR) criterion.

\section{Related Work}

\section{A. Opinion Mining}

Opinion mining, which is also referred as sentiment analysis, includes development of a system which able to assemble and classify opinions of a consumers about a product. 


\section{International Journal of Science and Research (IJSR) \\ ISSN (Online): 2319-7064}

Index Copernicus Value (2013): 6.14 | Impact Factor (2014): 5.611

Automated opinion mining often uses machine learning, a type of artificial intelligence (AI), for the purpose to mine text for sentiment.

Information available in text format can be categorized into facts and opinions. A fact represents the objective statements about entities and events where as opinions stand for subjective statements. Opinions imitate people's sentiments about the entities and events. Opinions provided by consumers in text reviews are examined from document, sentences included in that document and word and phrases included in that document [11]. Goal of such type of document-level (sentence-level) opinion mining is to categorize the overall subjectivity or sentiment expressed in an individual review document (sentence).

Evaluation of texts at the document or the sentence level does stands for the opinions of users such as likes and dislikes. A positive document does not represent the all positive opinions of consumers on features of particular object. Similarly, a negative document does not stand for all negative opinions of users on features of particular

Text document which includes evaluations holds both positive and negative aspects of particular object or entity according to user's views. Generally, overall sentiment on the object may contain some positive aspects and some negative aspects. Strong analysis of feature level is required to find complete aspects about object or entity. For this purpose three major tasks are as follows: 1) Identifying object features 2) Determining opinion orientations 3) Grouping synonyms

Identify object features search out for recurrent nouns and noun phrases as features, which are usually authentic features. Existing information extraction methods which are applicable for identifying object features are as conditional random fields (CRF), hidden Markov models (HMM). Determining opinion orientations conclude whether the opinions given by consumer on the features of object or entity are positive, negative or neutral. Existing lexiconbased approach uses opinion words and phrases in a sentence to decide the orientation of an opinion on a feature. One object features can be expressed with different words or phrases, grouping synonyms task groups synonyms together.

To calculate sentence subjectivity Hatzivassilglou and Wiebe [16] presents supervised classification technique to forecast sentence subjectivity. Hatzivassiloglou and Wiebe proposed the overall effects of dynamic adjectives, semantically oriented adjectives, and gradable adjectives on predicting subjectivity of the text document holding reviews. Pang and Lee [11] proposed a sentence-level subjectivity detector for the purpose to find out the sentences in a document as either subjective or objective. This technique retains subjective sentences and discards the objective sentences. After then they applied sentiment classifier. Task of sentiment classifier is to abstract resulted subjectivity with enhanced results.

To categorize entire movie reviews into positive or negative sentiments, Pang et al. [14] introduced machine learning model as naive Bayes, maximum entropy, and support vector machines. They conclude results generated by standard machine learning methods are superior to result by humangenerated baselines. But machine learning method performs well on only traditional topic based categorization and lack in functionality on sentiment classification.

An unsupervised learning method was proposed to categorize review documents into positive or negative in which as thumbs up represented positivity of document and thumbs down represents negativity of document [8].

Alculated of each review document to anticipate sentiment of review document. To compute sentiments of phrases in review document, domain-dependent contextual information is used but this technique has limitation as it depends on external search engine.

Zhang et al. [6] introduced a rule-based semantic analysis technique to categorized sentiments for text reviews. Word dependence structures are used to classify the sentiment of a sentence. Zhang et al. predicted document-level sentiments by aggregating sentiments of sentence. This technique possesses limitation as rule-based methods experience poor exposure as they do not hold comprehensiveness in their rules. To avoid this, Maas et al. [15] presented method for both document-level and sentence-level sentiment classification. This pro-posed method uses combination of unsupervised and supervised approaches to learn vectors. For learning process, they capture semantic term-document information as well as rich sentiment content.

It is essential to note that opinion mining of the document, sentence, or phrase (word) level does not determine what exactly people liked and disliked in reviews. It fails to combine the identified sentiments and equivalent features commented on in the reviews. Clearly, an extracted opinion without the corresponding feature (opinionated target) is of limited value in reality [2].

\section{B. Opinion Feature Extraction}

Opinion feature extraction is a subproblem of opinion mining. Existing techniques of opinion feature extraction can be categorized into two categories as, supervised and unsupervised.

To mark features or aspects of observed entities, supervised learning combines hidden Markov models and conditional random fields. This is also known as a joint structural tagging problem. Though supervised models perform well on given domain, they required extensive retraining when used in several domains. To use supervised models in different domains, transfer learning process s required.

Unsupervised Natural language Processing NLP methods utilize mining of syntactic patterns of features to abstract opinion features. Unsupervised approaches determine syntactic relations between feature terms and opinion words in sentences. To determine relations unsupervised approaches make use of crafted syntactic rules or semantic role labeling [10]. This relation assists to locate features related with opinion words as well as mine large number of invalid features of online reviews. For the purpose of extraction of frequent itemsets $\mathrm{Hu}$ and Liu [12] introduced an association 


\section{International Journal of Science and Research (IJSR) \\ ISSN (Online): 2319-7064}

Index Copernicus Value (2013): 6.14 | Impact Factor (2014): 5.611

rule mining $(\mathrm{ARM})$ technique which relies on frequency of itemsets. Frequent itemset consists of potential opinion features, which are nouns and noun phrases with high sentence-level frequency. But this technique has restrictions as: 1) frequent but invalid features are extracted incorrectly, and 2) rare but valid features may be overlooked.

$\mathrm{Su}$ et al. [8] proposed a mutual reinforcement clustering (MRC) technique to tackle feature-based opinion mining problems. Mutual reinforcement clustering methods are used to mine the relations between feature categories and opinion word groups. Extraction process depends on a cooccurrence weight matrix generated from the given review corpus. MRC also able to extract infrequent features if the mutual relationships between feature and opinion groups found through the clustering phase is accurate. MRCs accuracy is low as it has troubles in obtaining good clusters on real-life reviews.

\section{Proposed System}

The feature battery in mobile domain is domain specific as it has higher frequency in mobile domain than outside domain. This work identifies Nouns, Noun phrases and adjectives by applying part of speech tagging on user input review. The next step is the extraction of candidate features by application of syntactic rules on output of POST. The extraction of these domain specific candidate features is based on the designing of syntactic rules. Domain relevance score is measured on each domain dependent corpus called intrinsic domain relevance score and on domain independent corpus called extrinsic domain relevance score by application of IDR/EDR algorithm. The candidate features with IDR score greater than user defined intrinsic relevance threshold and EDR scores less than user defined extrinsic relevance threshold are the exact opinion features. These extracted features are more domain specific and less generic features. The identification of opinion features from candidate features is done by application of IEDR algorithm.

As shown in architecture diagram user select reviews from either internet in the form of html file or from text file on local system. The part of speech tagging is applied on the collected reviews for classification of nouns, noun phrases, or adjectives. The application of language dependent syntactic rules will find most probable features from the user review.

The features extracted in this phase may be incorrect; to filter irrelevant features intrinsic domain relevance and extrinsic domain relevance scores are measured on domain dependent corpus and domain independent corpus respectively. This domain relevance score represents frequency of relevant feature term in a specific document. The last step is the application of intrinsic extrinsic domain relevance, in IEDR two thresholds are selected called intrinsic relevance threshold and extrinsic relevance threshold. The features with IDR score greater than intrinsic relevance threshold and EDR score less than extrinsic relevance threshold are extracted as an opinion features. They are more domains specific and less generic.

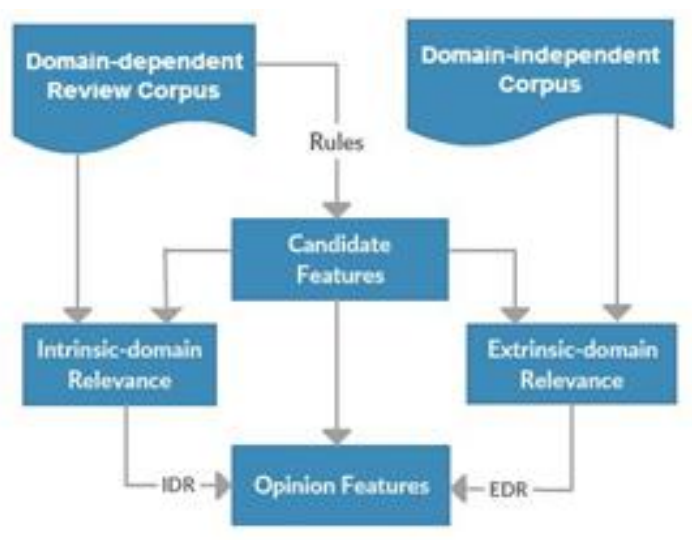

Figure 1: Architecture of the Proposed System

As shown in the figure the opinion feature price which is associated with adjective expensive. In other figure noun feature exterior associated with the verb like. The first step for extracting candidate feature is the construction of dependence tree. The second step is the application of the syntactic rules for candidate feature identification. As shown in table1 there are number of syntactic rules for extraction of candidate features from user review.

\section{Algorithm Used}

\section{A. Algorithms}

1) Calculation of Intrinsic/Extrinsic domain relevance Input: Domain specific/Independent corpus Output: Domain relevance score (IDR/EDR)

1. For each candidate feature in corpus $\mathrm{C}$ calculate wij.

2. Calculate standard deviation si.

3. Calculate Dispersion dispi.

4. Calculate Deviation deviij .

5. Calculate Domain relevance drij.

2) Identification of Opinion features using IEDR Input: Domain Review corpus $\mathrm{R}$ and Domain independent corpus D Output: A validated list of opinion features.

1. Find candidate features.

2. For each candidate feature calculate intrinsic domain relevance idri on review corpus $\mathrm{R}$.

3. For each candidate feature calculate extrinsic domain relevance edri on domain independent corpus D.

4. Candidate features with idr score greater than threshold vale and edr score less than another threshold are conformed as opinion features. 5) Calculate Domain relevance drij candidate features as a output to the user. This algorithm identifies opinion features by selecting two threshold values.

\section{B. Analysis of Algorithms}

Intrinsic Domain Relevance / Extrinsic Domain Relevance: The Intrinsic Domain Relevance and Extrinsic Domain Relevance Algorithms are NP complete type of problems, because they return domain relevance value and executes in polynomial time. These algorithms to find domain relevance values for input reviews. Intrinsic Extrinsic Domain Relevance: The Intrinsic Extrinsic Domain Relevance Algorithms are NP complete type of problems, because it executes in polynomial time and return candidate features as a output to the user. This algorithm identifies opinion features by selecting two threshold values. 


\section{Conclusion}

In this paper, we proposed a web crawler for fetching data/reviews from web pages and then opinion feature extraction based on the IEDR feature-filtering criterion, which utilizes the disparities in distributional characteristics of features across two corpora, one domain-specific and one domain-independent. For each extracted candidate feature, we then estimate its intrinsic-domain relevance (IDR) and extrinsic-domain relevance (EDR) scores on the domaindependent and domain-independent corpora, respectively. And then we call this interval thresholding approach the intrinsic and extrinsic domain relevance (IEDR) criterion.

We found that using a domain-independent corpus of a similar size as but topically different from the given review domain will yield good opinion feature extraction results.

\section{References}

[1] Zhen Hai, Kuiyu Chang, Jung-Jae Kim, and Christopher C. Yang, Iden-tifying Features in Opinion Mining via Intrinsic and Extrinsic Domain Relevance, IEEE Transactions on Knowledge and Data Engineering, Vol. 26, No. 3, March 2014

[2] Y. Jo and A.H. Oh, Aspect and Sentiment Unification Model for Online Review Analysis, Proc. Fourth ACM Intl Conf. Web Search and Data Mining, pp. 815-824, 2011.

[3] N. Jakob and I. Gurevych, Extracting Opinion Targets in a Singleand Cross-Domain Setting with Conditional Random Fields, Proc. Conf. Empirical Methods in Natural Language Processing, pp. 1035- 1045, 2010.

[4] F. Li, C. Han, M. Huang, X. Zhu, Y.-J. Xia, S. Zhang, and $\mathrm{H}$. $\mathrm{Yu}$, Structure-Aware Review Mining and Summarization, Proc. 23rd Intl Conf. Computational Linguistics, pp. 653-661, 2010.

[5] C. Zhang, D. Zeng, J. Li, F.-Y. Wang, and W. Zuo, Sentiment Analysis of Chinese Documents: From Sentence to Document Level, J. Am. Soc. Information Science and Technology, vol. 60 , no. 12, pp. 2474-2487, Dec. 2009.

[6] G. Qiu, C. Wang, J. Bu, K. Liu, and C. Chen, Incorporate the Syntactic Knowledge in Opinion Mining in User-Generated Content, Proc. WWW 2008 Workshop NLP Challenges in the Information Explosion Era, 2008.

[7] Q. Su, X. Xu, H. Guo, Z. Guo, X. Wu, X. Zhang, B. Swen, and Z. Su, Hidden Sentiment Association in Chinese Web Opinion Mining, Proc. 17th Intl Conf. World Wide Web, pp. 959-968, 2008.

[8] R. Mcdonald, K. Hannan, T. Neylon, M. Wells, and J. Reynar, Structured Models for Fine-to-Coarse Sentiment Analysis, Proc. 45th Ann. Meeting of the Assoc. of Computational Linguistics, pp. 432- 439, 2007.

[9] B. Pang and L. Lee, A Sentimental Education: Sentiment Analysis Using Subjectivity Summarization Based on Minimum Cuts, Proc. 42nd Ann. Meeting on Assoc. for Computational Linguistics, 2004.

[10]E. Cambria, D. Olsher, and K. Kwok, Sentic Activation: A Two- Level Affective Common Sense Reasoning Framework, Proc. 26th AAAI Conf. Artificial Intelligence, pp. 186-192, 2002. 